\title{
Evaluación de tres dietas con harina de hoja de bore (Alocasia macrorrhiza) en pollos de engorde
}

\section{Evaluation of three bore leaf meal (Alocasia macrorrhiza) diets in broilers}

\author{
Fredy López M, ${ }^{1 *}$ M.Sc, Alex Caicedo G, ${ }^{1}$ IA, Gustavo Alegría F, ${ }^{1}$ IA. \\ ${ }^{1}$ Universidad del Cauca. Facultad de Ciencias Agropecuarias, Popayan - Colombia. ${ }^{2}$ Ingenieros \\ Agropecuarios, Universidad del Cauca.*Correspondencia: fjlopez@unicauca.edu.co
}

Recibido: Marzo de 2010; Aceptado: Enero de 2012.

\section{RESUMEN}

Objetivo. Evaluar el efecto nutricional de incluir la harina de hoja de bore, en proporciones de 0 (T1), 5 (T2), 10 (T3) y 15\% (T4), en la alimentación de 100 pollos machos de la línea Ross 308. Materiales y Métodos. Se empleo un diseño experimental completamente al azar con cuatro tratamientos, cinco repeticiones por tratamiento y cinco unidades experimentales por repetición. Para su respectivo análisis se registró consumo diario, ganancia de peso, conversión alimenticia, mortalidad y eficiencia económica, durante un periodo de 6 semanas. Resultados. Se observó que no existieron diferencias estadísticas significativas en conversión alimenticia, pero si en consumo, ganancia de peso y eficiencia económica $(\mathrm{p}<0.05)$, siendo el mejor tratamiento el T2, con un consumo de $4003.4 \mathrm{~g}$, una ganancia de peso de $1794.4 \mathrm{~g}$ una conversión de 2.4 y un beneficio neto de campo de $107.88 \%$. Conclusiones. La harina de hoja de bore es una alternativa de alimentación para pollos de engorde, al emplearla al 5\% de nivel de inclusión, como materia prima no convencional, la cual permite generar un buen comportamiento productivo y económico.

Palabras Clave: Alimentos no convencionales, dieta, forraje (Fuente: CAB).

\begin{abstract}
Objective. Assessing the nutritional impact of including bore leaf meal in proportions of 0 (T1), 5 (T2), 10 (T3) and 15\% (T4) in the supply of 100 male chickens of the Ross 308 line. Materials and Methods. A completely randomized design with four treatments, five rounds per treatment and five experimental units per round was used. For the corresponding analysis, daily consumption, weight gain, feed conversion, mortality and economic efficiency, for a period of 6 weeks were recorded. Results. No statistically significant differences in feed conversion were observed, but differenced in consumption, weight gain and economic efficiency $(p<0.05)$; with T2 being the best treatment, with a consumption of $4003.4 \mathrm{~g}$, a weight gain of $1794.4 \mathrm{~g}$, a conversion of 2.4 , and a field net profit of $107.88 \%$ were established. Conclusions. The bore leaf meal is an alternative feed for broilers, when employed at $5 \%$ of inclusion level, as non-conventional raw material, which can generate a good productive and economic behavior.
\end{abstract}

Key words: Diets, forage, unconventional foods (Source: $C A B$ ). 


\section{INTRODUCCIÓN}

Los países desarrollados se caracterizan por su alto nivel de investigación dirigido a cultivos adaptados a su medio y a la búsqueda de nuevos renglones productivos para estos; es así como se manifiesta un alto nivel económico y productivo de estos países, los cuales presentan ventajas tecnológicas comparados con países como el nuestro. En Colombia, y en especial en el Municipio de El Tambo, muchas de las especies vegetales útiles como alternativas alimentarías en pollos de engorde, están subvalorados y no son explotados adecuadamente, siendo estos quizás la solución a la problemática de los altos costos de alimentación que se presenta en las explotaciones avícolas ( $70 \%$ de la producción) (1), en los cuales existe una alta dependencia de recursos alimenticios convencionales importados, que genera que el precio de las dietas, este sujeto a las fluctuaciones externas del mercado.

Por lo tanto, la Alocasia macrorrhiza comúnmente conocida con el nombre de bore, se constituye en un recurso alimenticio potencial, como complemento a las propuestas de utilización de recursos convencionales, en la alimentación de pollos de engorde; es una especie herbácea perenne que puede llegar a los $5 \mathrm{~m}$ de altura, presenta un alto grado de adaptabilidad para las condiciones medioambientales del trópico bajo y medio, con suelos de baja fertilidad y con un excelente valor nutritivo (2).

El presente estudio evaluó la inclusión de harina de hoja de bore (Alocasia macrorrhiza) en la alimentación de pollos de engorde, como fuente parcial de proteína, en niveles de 5,10 y $15 \%$, de materia seca (MS) de la dieta, teniendo como variables de respuesta la ganancia de peso, consumo de alimento, conversión alimenticia y otros aspectos como mortalidad, factor de eficiencia europeo (F.E.E.) y rentabilidad en la etapa de iniciación y finalización.

\section{MATERIALES Y MÉTODOS}

Área de estudio. El proyecto se realizó en La Corporación Maestra Vida, ubicada en el Municipio de El Tambo - Cauca, a 25 kilómetros al suroccidente de la ciudad de Popayán, con coordenadas geográficas: $2{ }^{\circ} 27^{\prime} 15^{\prime \prime}$ latitud norte, $76^{\circ} 49^{\prime} 1^{\prime \prime}$ longitud este, a una altura 1750 msnm, temperatura de $18{ }^{\circ} \mathrm{C}$ y precipitación promedio anual de $2800 \mathrm{~mm}$ (3).

Instalaciones. Se trabajó en un galpón de $27 \mathrm{~m}^{2}$, sobre piso en tierra, techo en zinc, paredes en esterilla y malla, dentro del cual se diseñaron 20 divisiones de $1 \mathrm{~m}$ de largo por $0.5 \mathrm{~m}$ de ancho con el fin de alojar 5 pollos por división en relación de 10 pollos $/ \mathrm{m}^{2}$.

Muestra. Se utilizaron 100 pollos machos de la línea Ross 308 de un día de nacidos, estos se sometieron a una valoración de calidad, en la cual se incluyó un pesaje inicial y aspectos fenotípicos como color del plumón, picos, ombligo seco, tamaño y fortaleza de patas. La fase experimental duró 42 días representados en 6 semanas, ( 3 semanas para cada etapa, iniciación y finalización respectivamente).

Suministro de alimento. Las diferentes dietas, acordes a los requerimientos nutricionales del ave, fueron repartidas en dos suministros al día, el primero en la mañana (7:00 a.m.) y el segundo en la tarde (4:00 p.m.) la cantidad suministrada fue de acuerdo con la tabla de consumo comercial usada en la zona de estudio. El alimento rechazado se recogía y se pesaba diariamente, para determinar el consumo real por parte de los animales evaluados, al igual que se efectuaron pesajes semanales antes de suministrar la primera ración.

Las variables evaluadas fueron ganancia de peso, consumo, conversión alimenticia y mortalidad, en cada etapa productiva.

Análisis estadístico. Se utilizó un diseño completamente al azar con cuatro tratamientos, cinco repeticiones y cinco unidades pollos por repetición; la diferencia entre tratamientos se basó en los niveles de inclusión de harina de hoja de bore (Alocasia macrorrhiza ), siendo el T1: el testigo (concentrado no convencional), T2, T3 y T4 concentrados con 5,10 y $15 \%$ de harina de hoja de bore, respectivamente, mas la utilización de materias primas como torta de soya, maíz, harina de pescado, aceite de girasol, harina de huesos, carbonato de calcio, coccidiostato, sal común y premezcla; dichos niveles de inclusión partieron de la base del preensayo efectuado en el Centro Latinoamericano de Especies Menores (Clem - Tuluá), en el año 2007.

Cada división representa una repetición que se distribuyó al azar, en estas se adecuó un comedero y un bebedero, buscando el suministro adecuado de cada dieta. Los resultados obtenidos se evaluaron por medio de análisis de varianza y pruebas de comparación múltiple de Duncan.

Análisis económico. Para contrastar al tratamiento control con los demás tratamientos y determinar su viabilidad en términos 
económicos, se empleó la metodología de presupuestos parciales, que permite organizar la información experimental de tal manera que ayude a tomar una decisión, sobre el tratamiento más conveniente (4). Mientras que el desempeño productivo de cada tratamiento se midió por medio del factor de eficiencia europeo (F.E.E.).

\section{RESULTADOS}

Consumo de alimento etapa de iniciación y finalización. El análisis de varianza permite evidenciar, que existieron diferencias significativas entre los tratamientos $(p<0.05)$, para ambas etapas productivas. Con respecto a la etapa de iniciación, la prueba de Duncan, mostró que se formaron tres grupos, uno por el tratamiento de mayor ganancia de peso (T1), un segundo grupo con T2, y un tercer grupo con T3 y T4, siendo estos últimos, los que reportan menor consumo con 867.2 y $839.5 \mathrm{~g}$, respectivamente (Figura 1 ).

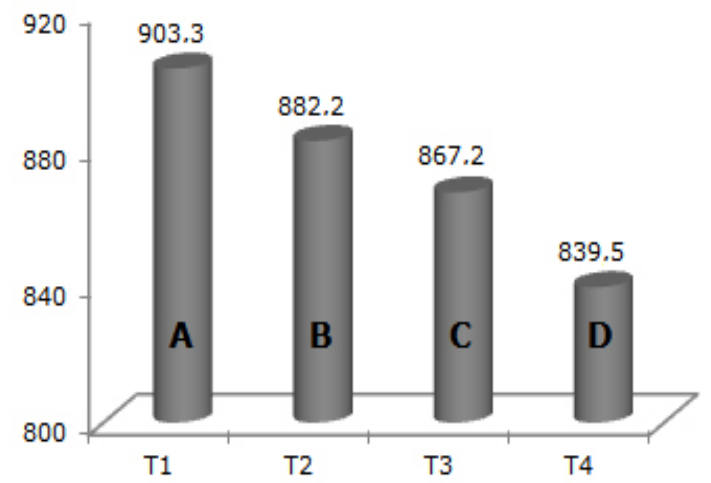

Figura 1. Consumo de alimento etapa de iniciación.

Con respecto a la etapa de finalización, la prueba de Duncan determinó que todos los tratamientos fueron diferentes entre sí, siendo el tratamiento testigo (T1) el que más alimento consumió $(3136 \mathrm{~g})$, mientras que los tratamientos T2, T3 y T4 los de menor consumo con 3121.3, 3091.5 y $3038.6 \mathrm{~g}$, respectivamente (Figura 2).

Ganancia de peso etapa de iniciación y finalización. Para esta variable en etapa de iniciación, no se encontraron diferencias significativas entre los tratamientos ( $p>$ 0.05 ), siendo el T2 el de mayor ganancia con $488 \mathrm{~g}$, contra $397.6 \mathrm{~g}$ para el T4 que presentó el menor valor; caso contrario a la etapa de finalización, donde si se encontraron diferencias estadísticas $(p<0.05)$ entre los tratamientos,

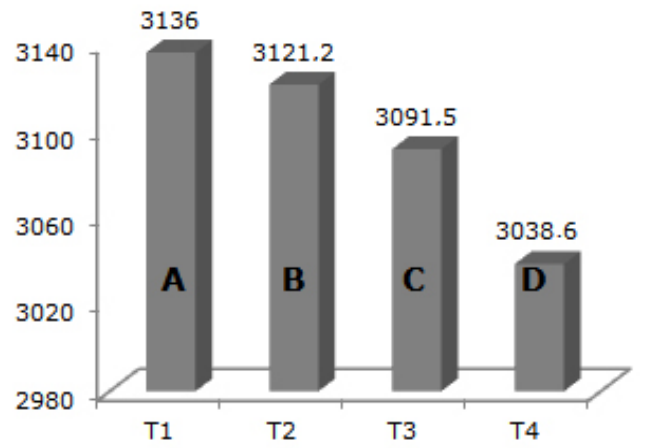

Figura 2. Consumo de alimento etapa de finalización.

donde se formaron tres grupos, los grupos 1 y 2 conformados por los tratamientos T2 y $\mathrm{T} 1$, respectivamente y el grupo tres con los tratamientos T3 y T4, quienes presentaron las menores ganancias de peso con 1620.6 y $1527.4 \mathrm{~g}$, respectivamente (Figura 3 ).

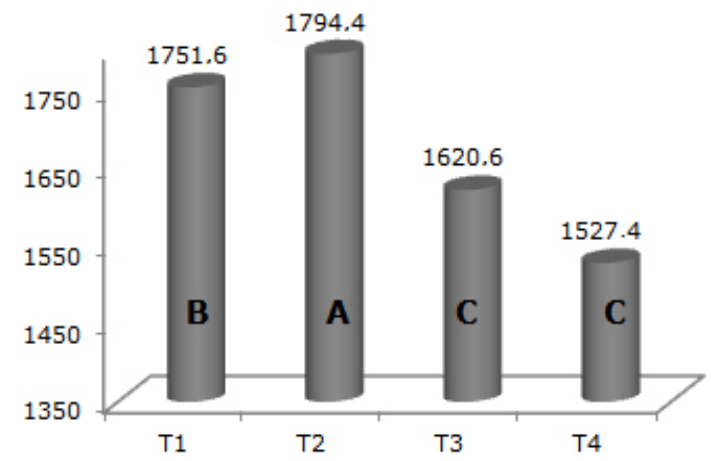

Figura 3. Ganancia de peso etapa de finalización.

Conversión alimenticia etapa de iniciación y finalización. Para ambas etapas no se obtuvieron diferencias estadísticamente significativas entre los tratamientos ( $p>0.05)$, siendo el T2 el que obtuvo la mejor conversión tanto en iniciación como finalización con 1.81 y 2.40, respectivamente (Figura 4 ).

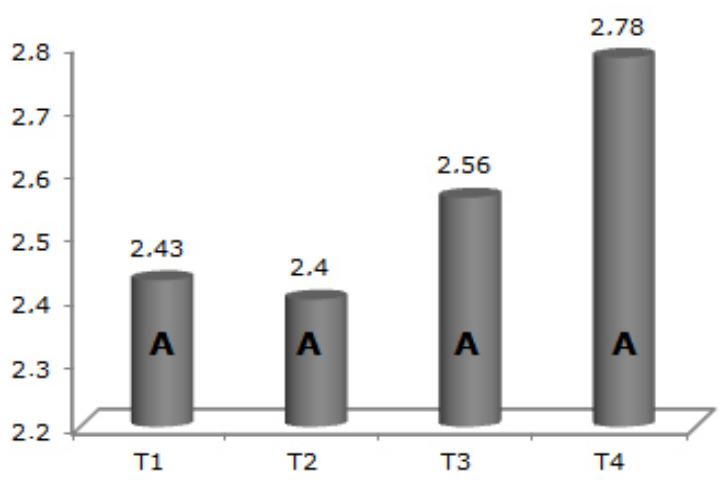

Figura 4. Conversión alimenticia etapa de finalización. 
Mortalidad. El índice de mortalidad fue de $0 \%$ en todos los tratamientos evaluados durante el ensayo.

Análisis económico. La relación costo del alimento y consumo de cada tratamiento (Tabla 1), permite evidenciar que una mayor inclusión de harina de hoja de bore, permite obtener reducciones en los costos variables, siendo el T4 el que mayor reducción presenta con un porcentaje de $14.15 \%$ frente al tratamiento testigo.

Tabla 1. Beneficio Bruto y Neto de campo por tratamiento.

\begin{tabular}{cccccc}
\hline Tto & $\begin{array}{c}\text { B.B.C. } \\
\mathbf{( \$ )}\end{array}$ & C.V. $\mathbf{( \$ )}$ & $\begin{array}{c}\text { B.N.C. } \\
\mathbf{( \$ )}\end{array}$ & $\mathbf{( \% )}$ & $\begin{array}{c}\text { Reducción } \\
\text { Costos } \\
\mathbf{( \% )}\end{array}$ \\
\hline T1 & 236.4 & 98.7 & 137.6 & 100.0 & 0.0 \\
T2 & 242.2 & 93.7 & 148.5 & 107.8 & 5.1 \\
T3 & 218.7 & 89.2 & 129.4 & 94.0 & 9.1 \\
T4 & 206.1 & 84.7 & 121.4 & 88.1 & 14.1 \\
\hline
\end{tabular}

Beneficio bruto de campo; CV = Costos variables; BNC = Beneficio Neto de Campo.

Las dietas experimentales T3 y T4 no reunieron las características necesarias para sustituir al concentrado testigo ya que a pesar de ser más económicas, no son rentables, pues estos tratamientos generan menor ganancia de peso y no compensa la reducción en el costo de alimentación. Mientras que el tratamiento 2 genera $7.8 \%$ más rentabilidad que el concentrado testigo (Tabla 1), permitiendo afirmar que niveles de $5 \%$ de hoja de bore en raciones para alimentación de pollos de engorde, aporta los nutrientes necesarios en la producción de esta especie a un bajo costo.

Factor de eficiencia europeo. En términos generales, el factor de eficiencia europeo para cada tratamiento reporta valores bajos, indicando que el lote en general no fue eficiente; sin embargo al comparar los tratamientos evaluados (T2, T3 y T4) con valores de 178.3, 150.5 y 130.9 respectivamente, contra el testigo 171.5 (T1), se puede determinar que el tratamiento que mejor desempeño obtuvo durante los 42 días del ensayo fue el T2, mientras que el T4, fue el de menor desempeño productivo.

\section{DISCUSIÓN}

El consumo de alimento se puede ver influenciado sustancialmente por muchos factores incluyendo el manejo de la parvada, la calidad del alimento, el estado de salud y las condiciones climáticas (5). Es importante aclarar, que las condiciones de manejo, sanidad y ambiente fueron similares para cada tratamiento.
De igual forma es importante mencionar, que el balance dispuesto para cada tratamiento (Tabla 2), buscaba suplir los requerimientos nutricionales necesarios para un optimo desarrollo fisiológico de los pollos en cada una de las fases; sin embargo al adicionar los tres niveles de inclusión de harina de hoja de bore, se generó un aumento de la fibra; siendo el tratamiento testigo (T1) el de menor concentración de esta $(3.15 \%)$ y los tratamientos T2, T3 y T4 los de mayor nivel con $3.6,4.04,4.48 \%$ respectivamente, para la etapa de iniciación. Esta diferencia en el balance de las dietas, genera disminución en el consumo presentado por los tratamientos con mayor proporción de bore, ya que excesos de fibra en la ración ( $>4 \%$ ) causan efectos negativos sobre la productividad en monogástricos jóvenes, relacionado con la palatabilidad, reducción de digestibilidad de los nutrientes y sensación de saciedad, haciendo que el pollo sea incapaz de consumir la cantidad de alimento necesaria, para cubrir sus necesidades energéticas (6).

Tabla 2. Balance nutricional por tratamiento y etapa productiva.

\begin{tabular}{ccccccc}
\hline Etapa & \multicolumn{3}{c}{ Iniciación } & \multicolumn{3}{c}{ Finalización } \\
T \% & M & L & F (\%) & M & L & F (\%) \\
\hline T1 0 & 0.4 & 1.0 & 3.15 & 0.34 & 0.8 & 2.89 \\
T2 5 & 0.4 & 0.99 & 3.6 & 0.35 & 0.79 & 3.34 \\
T3 10 & 0.4 & 0.98 & 4.04 & 0.35 & 0.78 & 3.37 \\
T4 15 & 0.4 & 0.97 & 4.48 & 0.6 & 0.77 & 4.21 \\
Req & 0.5 & 1.1 & 4 & 0.35 & 0.85 & 4 \\
\hline
\end{tabular}

Metionina; L=Lisina; F= Fibra

Este comportamiento fisiológico, es acorde a lo reportado por González et al (7), quienes al trabajar con niveles de 0 hasta $10 \%$ de inclusión de harina de hojas de bore, encontraron disminución en el consumo, en la medida que el nivel de inclusión aumentaba. De igual forma , otros autores (8), reportan disminución en el consumo de alimento en dietas donde se utilizo 15 y $30 \%$ de nivel de inclusión de Canavalia brasilensis, en dietas para pollos de engorde, argumentando de igual forma, los efectos generados por el incremento de la fibra acorde al nivel de inclusión de este forraje.

En el mismo contexto, otros autores (9), manifiestan que el hambre esta relacionada con una necesidad fisiológica, y el apetito relacionado con diferentes tipos de motivación, como un deseo de repetir una experiencia placentera; sin embargo, estas se ven afectados por factores como la palatabilidad, capacidad física del tracto digestivo y la voluminosidad de la dieta. Debido a su volumen, un ingrediente puede restringir el consumo de alimento, si 
la cantidad de alimento que el animal debiera comer para llenar sus requerimientos, es mayor que su capacidad física que lo permita; igualmente, la distensión del buche de los pollos con alimento húmedo o con alto contenido de fibra, deprimen el consumo durante las siguientes 3 horas, ya que hay señales físicas que trasmiten receptores dentro del buche. Estos receptores son sensibles a la presión a la que son sujetos. Una vez que se perciben, los mensajes que se envían al cerebro se integran a la señal de saciedad, reduciendo así el consumo de alimento. Particularmente en aves, factores como el apetito específico por aminoácidos y minerales hace que un déficit oexceso moderado de estos nutrientes, provoque un aumento o una disminución de la ingestión del alimento.

No obstante, cualquier deficiencia grave en nutrientes esenciales causa una drástica reducción del consumo, por ejemplo, un bajo contenido proteico o un desequilibrio importante de aminoácidos (9) y por lo tanto, bajo condiciones comerciales donde solo hay una opción de alimento balanceado disponible, el consumo de alimento está marcadamente influenciado tanto por el perfil energético de la dieta, como por el de aminoácidos.

Por otra parte, pese a que no existió diferencia de ganancia de peso entre los tratamientos; estos valores son bajos comparados con los reportados por la tabla de ganancia de peso de la línea Ross 308, la que sugiere que un pollo de 21 días debe de estar pesando en promedio $755 \mathrm{~g}$, peso que no fue alcanzado por ninguno de los tratamientos evaluados.

Este efecto se podría atribuir a la deficiencia en lisina (T1:1\%, T2:0.99\%, T3:0.98\% y $\mathrm{T} 4: 0.97 \%)$, aminoácido que tienen un papel muy importante en las dos primeras semanas y puede influir en el desempeño final del ave y en el rendimiento de la canal.

En comparación con otros aminoácidos, el contenido de lisina es relativamente alto en la carne de pechuga y considerando que este músculo representa el $21 \%$ de la canal en pollos de 7 días y $27 \%$ en pollos entre los 7 y 14 días de edad; por lo que, proporcionar una cantidad adecuada de lisina ( 1.11 y $1.21 \%)$, durante las dos primeras semanas del crecimiento puede incrementar el rendimiento de la pechuga y en el peso al mercado (8).

Es importante destacar, que esta experimentación buscó determinar el desempeño productivo y económico del lote, bajo un sistema de alimentación no convencional, sin la necesidad de incurrir en insumos externos y altamente costosos, como lo son los aminoácidos sintéticos (Lisina y Metionina), los cuales son aportados por los ingredientes de la dieta y son cercanos al requerimiento del ave. Por otra parte, la genética avícola moderna, permiten que los pollos de engorde incrementan su peso 50 veces desde el nacimiento hasta llegar al mercado en un periodo de 6 semanas (10-12), permitiendo inferir que la nutrición adecuada contribuyen a este alto nivel de desarrollo actual. Sin embargo, el manejo deficiente o una nutrición subóptima cuando la parvada es joven, pueden tener un importante efecto en el peso al mercado. Las aves no pueden recobrar completamente la pérdida de peso en una etapa temprana y por lo tanto se reflejara en el peso al sacrificio (10), esto se presentó durante esta evaluación; los pollos en general no terminaron con un buen peso en la etapa de iniciación y por lo tanto, iniciaron con bajos pesos en la etapa de finalización, propiciando bajos rendimientos al sacrificio, efecto más marcado en los tratamientos 3 y 4 ( 10 y $15 \%$ de bore), lo cual es consonante a un mayor nivel de fibra, lo que afecta el consumo y por ende su ganancia de peso, tal como lo reportan diversos autores (13), al evaluar diferentes niveles de inclusión de morera morus alba, con niveles de inclusión de 5, 10 y $15 \%$, y otros reportes (14), al evaluar niveles de inclusión de 15 y 30\% de Vigna unguiculata, encontrando que a medida que se aumenta la inclusión de estos follajes, se disminuye la ganancia de peso.

Parvadas que exhiben los promedios más altos de ganancia de peso casi siempre tienen los consumos más altos de alimento y frecuentemente tienen las mejores conversiones alimenticias y tasas de viabilidad (10). Basado en esto, se puede determinar que posiblemente la diferencia de peso que se presentó en esta fase, se debe al bajo consumo de alimento observado por los tratamientos T3 y T4 comparado con los otros dos tratamientos, que generó un atraso significativo en el desarrollo de los animales.

De igual forma, las pruebas de ganancia de peso, se mencionan como la metodología por excelencia para medir la biodisponibilidad de nutrientes, ya que para obtener una respuesta en la ganancia de peso, es necesario que los componentes de la dieta sean digeridos, absorbidos y utilizados para las funciones de mantenimiento, crecimiento y/o producción (15) y por lo tanto al comparar el tratamiento 1 y 2 se considera que la diferencia entre las dos raciones se debió posiblemente, a una mayor digestibilidad del concentrado a evaluar 
con relación al testigo. Esto se debe a que los niveles de $5 \%$ de inclusión de harina de hoja de bore, mantuvieron niveles más bajos de fibra en comparación a los otros tratamientos experimentales y por lo tanto, permitió reflejar el potencial de este material en el desarrollo de la parvada bajo esta dieta.

Por otra parte, el índice de conversión se define como la relación entre la cantidad de alimento consumido y la producción (ganancia de peso), para este ensayo, se pudo determinar que no se presentaron diferencias estadísticas entre tratamientos. Sin embargo el T2 ostentó un índice de conversión 0.18 menos que el T1; 0.38 menos que el T3 y 0.42 menos que T4. Estos datos reflejan la importancia de la implementación de niveles bajos de bore en la dieta; los animales del tratamiento 2 fueron más eficientes y requirieron menos alimento para obtener una producción similar al tratamiento testigo (T1).

Sin embargo, la conversión obtenida para cada tratamiento es relativamente alta comparada con la reportada por la tabla de conversión alimenticia de la línea Ross 308, la cual indica que para la etapa de finalización, se debe manejar una conversión alimenticia de 1.89 aproximadamente, mientras que el tratamiento con conversión más cercano a este valor, es el tratamiento 2 (2.4), seguido por el 1,3 y 4 con $2.43,2.56$ y 2.78 respectivamente. Estos valores se asemejan a los obtenidos en otros ensayos (13), quienes al evaluar diferentes niveles de inclusión de morera 5, 10 y $15 \%$, obtuvieron valores de $2.34,2.67$ y 3.21 respectivamente.
Finalmente, la presentación del alimento para este ensayo (harina), posiblemente fue un factor adicional que pudo incidir en la alta conversión que presentó la parvada en general. Algunos autores $(16,17)$, reportan que en general, la forma física del alimento, harina o pellets, interviene en el desarrollo del tracto gastrointestinal (TGI); los alimentos en forma de pellets, tienen la ventaja de disminuir el tiempo de consumo y el ahorro de energía metabolizable; así, alimentos en diferentes tipos de pellets, como los crumbles, son ideales para los alimentos iníciales.

En conclusión, la harina de hoja de bore es una alternativa de alimentación para pollos de engorde, al emplearla al 5\% de nivel de inclusión, como materia prima no convencional, la cual permite generar un buen comportamiento productivo en términos de ganancia de peso, consumo y conversión, al igual que su reflejo en términos de una mayor rentabilidad al productor, debido a la disminución de la dependencia de algunos insumos externos de uso convencional, lo cual se traduce en la reducción en los costos de producción en un $5.13 \%$.

\section{Agradecimientos}

A las directivas de la Corporación Maestra Vida, por permitir el uso de sus espacios y el aporte del material forrajero para esta evaluación, al igual que a la Facultad de Ciencias Agropecuarias de la Universidad del Cauca.

\section{REFERENCIAS}

1. FENAVI. Boletín Fenaviquin. Noticias de Interés. [en línea] 2006 [fecha de acceso 23 de marzo de 2006]; URL disponible en : http:/www.fenavi.org

2. Gómez E. Una revisión sobre el Bore (Alocasia macrorrhiza). [en línea] 2006 [fecha de acceso 23 de marzo de 2007]; URL disponible en : http://www.fao.org/ Docrep/

3. [Mapa topográfico]. Colombia: Instituto Geográfico Agustín Codazi, 2008.
4. López Molina F. Suplementación con morera (Morus alba) para vacas Holstein en lactancia en la meseta de Popayán. [Tesis maestría].Colombia. Universidad Nacional de Colombia, sede Palmira; 2002.

5. Manual de manejo del pollo de engorde. Ross Breeders Limited. [en línea] 2003 [fecha de acceso 19 de abril de 2008]; URL disponible en: http://www.aviagen.com/ manual/ 
6. Mateos G., Lazaro J.M. y González E. Efecto de la fibra dietética en piensos en iniciación para pollitos y lechones. Departamento de producción animal. Universidad Politécnica de Madrid. [en línea] 2006 [fecha de acceso 21 de febrero de 2007]; URL disponible en: http://www.etsia.upm.es/fedna

7. González, C; Ruiz, D; Ariza, C; García, C; Evaluación nutricional y efecto pigmentante de la harina de hoja de Bore en pollos de engorde. Corporación Colombiana de Investigaciones Agropecuarias-ORPOICA. 2002 Pág: 31-32

8. Dorado E; Bravo S; Vivas N. Evaluación de dos niveles de inclusión de forraje de Canavalia brasilensis como reemplazo de la proteína de torta de soya en alimentación de pollos de engorde. Tesis pregrado. Colombia. Universidad del Cauca. 2012

9. Gernat A. Consumo de Alimento de Pollo de Engorde de A a la Z, Escuela Agrícola Panamericana (Zamorano). Honduras. [en línea] 2006 [fecha de acceso 15 de noviembre de 2007]; URL disponible en: http://www.engormix.com/consumo_ alimento_pollo_engorde.

10. Dozier A. Influencia de la nutrición temprana en el pollito: Revista Industria Avícola. (Atla) 2004; 112 (51).

11. Manual Nutrición Animal. La Ingestión de alimentos. [en línea] 2004 [fecha de acceso 11 de mayo de 2009]; URL disponible en: http://www.conejosyalgomas. com.ar

12. Ben M. Evaluación del complejo enzimático en la mejora del valor nutritivo de cereales y leguminosas en la alimentación de pollos en crecimiento. [en línea] 2004 [fecha de acceso 4 de abril de 2007]; URL disponible en : http://www.tdcat.cesca.es
13. Casamachin ,M; Díaz, D. Evaluación de tres niveles de inclusión de morera Morus alba en alimento para pollos de engorde. Trabajo de pregrado. Universidad del Cauca.

14. Calderón, L; Rengifo, S. Evaluación de niveles de inclusión de forraje de caupi Vigna unguiculata como reemplazo de la proteína de torta de soya en la alimentación de pollos de engorde. Trabajo de pregrado. Universidad del Cauca. 2011

15. Fernandez R.Biodisponibilidad de nutrientes en aves de distintas edades. [en línea] 2005 [fecha de acceso 22 de mayo de 2007]; URL disponible en : http://www. engormix.com/articulo_biodisponibilidad_ nutrientes_a_fu

16. Araque A, Argenti P, Páez L, Sánchez A, Sandoval E. Aspectos básicos para una explotación exitosa de pollos de engorde. [en línea] 2002 [fecha de acceso mayo de 2006]; URL disponible en:http:// www.cuencarural.com/granja/avicultura/ aspectos-basicos para una-explotacionexitosa-de-pollos-de-engorde/

17. Cáceres C, Cedeño L, Taylor S. Elaboración y evaluación de una ración alimentaria para pollos de engorde en un sistema bajo pastoreo con insumos del trópico húmedo. Costa Rica. [en línea] 2005 [fecha de acceso 13 de mayo de 2006]; URL disponible en : http://usi.earth. ac.cr/tierratropical/archivosdeusuario/ Edicion/27_v2.203_CaceresCedeno.pdf 